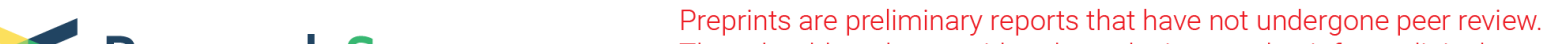 Research Square
or referencend by the media as validated information.
}

\section{The Small Molecule Components of Human Synovial Fluid and Bovine Calf Serum that Correlate with Cobalt Chrome (CoCrMo) Wear}

\section{Matthew Jaggard}

Imperial College of Science Technology and Medicine: Imperial College London

Harriet Stevenson

Imperial College of Science Technology and Medicine: Imperial College London

Claire Boulange

Imperial College of Science Technology and Medicine: Imperial College London

\section{Goncalo Graca}

Imperial College of Science Technology and Medicine: Imperial College London

\section{Pouya Akhbari}

Imperial College of Science Technology and Medicine: Imperial College London

\section{Uddhav Vaghela ( $\square$ uv14@ic.ac.uk)}

Imperial College of Science Technology and Medicine: Imperial College London https://orcid.org/00000003-3525-4388

\section{Rajarshi Bhattacharya}

Imperial College of Science Technology and Medicine: Imperial College London

Horace Williams

Imperial College of Science Technology and Medicine: Imperial College London John Lindon

Imperial College of Science Technology and Medicine: Imperial College London

Philippa Cann

Imperial College of Science Technology and Medicine: Imperial College London

Chinmay Gupte

Imperial College of Science Technology and Medicine: Imperial College London

\section{Research Article}

Keywords: Human Synovial Fluid (HSF), Nuclear Magnetic Resonance (NMR), 25\% wt Bovine Calf Serum (25BCS), metabolomics, Glycosaminoglycans (GAG)

Posted Date: September 24th, 2021

DOI: https://doi.org/10.21203/rs.3.rs-921974/v1 
License: (c) (i) This work is licensed under a Creative Commons Attribution 4.0 International License. Read Full License 


\section{Abstract}

\section{Background:}

Implant wear in joint replacements is influenced by the chemical and physical properties of human synovial fluid (HSF). In vitro testing for implant wear uses $25 \%$ weight bovine calf serum (25BCS) to substitute for HSF, due to similar rheology and total protein content. However, previous studies have shown differences in the macromolecular composition. We aimed to evaluate the differences in small molecule composition between fluids and correlate these differences with their effects on implant material wear.

\section{Methods:}

HSF was harvested from osteoarthritis patients undergoing primary knee replacement $(n=14)$. Nuclear magnetic resonance (NMR) spectroscopy with linear regression modelling analysed the metabolites present in HSF and commercially sourced 25BCS and investigated the differences. Wear properties of the fluids were evaluated using a validated quantitative laboratory bench-test utilising a cobalt/chromium/molybdenum (CoCrMo) ball oscillating against a CoCrMo disc and analysing the resulting wear scar. The variation in metabolite levels in both HSF and 25BCS was correlated to the wear properties of the fluids.

\section{Results:}

Differences in the levels of metabolites, lipids, and glycosaminoglycans (GAG) were observed between HSF and 25BCS: significance was confirmed by 0-PLS-DA ( $p<0.05)$. The wear of CoCrMo was found to strongly correlate with the macromolecules GAG and proteins that potentially bind to glucose and citrate.

\section{Conclusions:}

The small molecule concentration differences between the fluids questions the validity of 25BCS as a model for wear analysis. The demonstration of variable metabolites present in HSF which correlate with material wear has implications for implant failure and targeted therapeutic manipulation of these metabolites.

\section{Trial Registration:}

Ethical approval was granted by the NRES Committee London, Chelsea, UK on the 12th May 2015. The study was performed in accordance with the ethical standards in the 1964 Declaration of Helsinki.

\section{Background}

In excess of 160000 arthroplasty operations are performed annually in the United Kingdom, with some 14000 being revision procedures(1). Prosthetic joints are engineering systems; combining artificial 
materials implanted in a biological environment and lubricated by human synovial fluid (HSF). Excessive wear of the load-bearing surfaces which can be physical (due to acetabular edge-loading or high friction) or corrosive (due to chemical reaction of the implant surface with its environment) contributes to implant failure(2). Wear occurs when the lubricating film present in the sliding interface is too thin or unstable to separate and protect the bearing surfaces.

\section{Prosthesis testing and validation}

Previously implant wear was measured in joint simulators which replicate the motion and variable forces within a natural joint cycle(3). The prosthesis was subjected to a million cycles before wear measurement $(4,5)$. The testing occurred in an enclosed space, but with non-physiological lubricant volumes thereby diluting any products. Furthermore, 25BCS is a surrogate for HSF due to presumed similar total protein content and non-Newtonian rheological properties despite significant differences between the fluids(6). Joint simulators are preferred for validation but suffer poor reproducibility and need volumes in excess of $300 \mathrm{ml}$ (impossible with HSF)(7).

Many practicioners assume that joint simulators will behave consistently between different lubricants. This overlooks the differing interaction between bearing surfaces and lubricants and the impact of the lubricant chemistry, something lost when the fluid is constantly recycled(8). Our previous work has demonstrated the behaviour of the fluids to be inconsistent in bench-top wear testing(9).

A bench device is required to evaluate HSF lubrication since a joint simulator will not allow for the small sample samples(9). This test replicates contact pressures, kinematics and sliding speeds $\left(0-42 \mathrm{mms}^{-1}\right)$ experienced over the load-bearing part of the gait cycle for a weight bearing joint. The resultant initial contact pressure (mean $200 \mathrm{MPa}$ ) is higher than quoted for the peak load during walking gait cycle but represents a "jogging" gait thereby mirroring a severe wear condition $(10,11)$.

\section{Potential differences in lubricating fluid chemistry}

Synovial fluid is highly proteinaceous, containing inflammatory proteins, albumin and proteoglycans such as lubricin. It contains water, significant levels of lipid, cells and glycoprotein debris(12). In the metal on metal (CoCrMo/CoCrMo) prosthetic joint it is assumed that macromolecules bind to the implant surface providing boundary lubrication but the small molecule effects are unknown(6). Population variance of synovial fluid composition will affect implant longevity and may partly explain individual differences where excess wear is observed(13). This was evident in CoCrMo hip prosthesis failures, where a proportion of patients reported good results with no excessive metalosis and specific patient cohorts e.g, women, had higher failure rates(14).

Key differences exist between 25BCS and HSF both in the diseased and disease-free scenario(9). 25BCS contains albumin as the dominant protein, whereas HSF contains large amounts of proteoglycan complexes(15). Furthermore, in disease and in osteoarthritis, HSF contains elevated levels of proteoglycan complexes: hyaluronan and chondroitin sulphate(16). Both fluids contain lipids with similar 
relative amounts, although the lipid compositions are likely to be very different, as shown in other species(17). This could impact upon the fluid's lubricant properties since the species and organisation of these lipids impacts boundary film behaviour(18). Current joint implant wear testing incorporates the material properties but not the differing joint fluid chemistry(5). Lubricating fluid and the impact upon implant wear

There are multiple studies which have examined the how fluid components affect wear. These all involve the use of model fluids (like BCS) and the supplementation of various components, concentrating solely upon the macromolecules $(6,15,19-22)$. There are no studies which examine the wear of non-model HSF related to its composition. Furthermore, there are no studies directly comparing the small molecule content of the fluid (HSF or its model BCS) and the subsequent effect upon wear(8).

\section{Role of Hyaluronic Acid}

Hyaluronic acid (HA) is an abunant glycosaminoglycan which forms a component of the proteoglycan complex within synovial fluid. As such it has a role of attracting water and providing viscoelastic properties to the fluid. It has a clinical role in viscosupplementation in clinical practice, although the efficacy of this intervention is a matter of debate. Supplementation of HA has been used in simulated joints to reduce friction. It is unclear if this molecule is key to wear. Furthermore, the composition and structure of HA is not uniform, changeable and may be significant. We hope that more detailed information of structure may provide greater insight into which aspects of composition may play a role.

\section{Hypothesis}

We hypothesise that small molecule constituents within synovial fluid, either free or complexed to macromolecules will affect implant wear.e aim to study harvested HSF against BCS directly to describe the differences in small molecule composition whilst understanding which components are important in prosthetic wear.

\section{Metabolic profiling: describing the small molecules and metabolism}

Metabonomics, or metabolic phenotyping is defined as "the quantitative measurement of the dynamic multiparametric response of a living system to pathophysiological stimuli or genetic modification"(23). It is a powerful method to describe the complex small molecule composition of biofluids ( $\angle 4 \mathrm{kDa})$ and has been successfully used in clinical medicine, toxicology, environmental science and plant science(24-29).

NMR spectroscopy analysis of HSF has been used to provide a qualitative representation of the macromolecules and a comprehensive quantitative representation of the small molecules(29). As well as providing an overall profile using a pulse sequence commonly abbreviated as NOESYPR1D, spectra can be acquired using a Carr-Purcell-Meiboom-Gill (CPMG) pulse sequence, which attenuates the macromolecule signals, thereby enhancing the small molecule detection (details of these methods can be found(30)). To aid interpretation of the complex data sets and identify metabolites which are statistically 
significantly different between sample classes, multivariate linear regression models are employed. Metabolites are identified from spectral peaks using a mixture of library referencing and analytical confirmation. We aim to use this method to provide a detailed small molecule description of the biofluids.

\section{Methods}

\section{Sample collection}

Two batches of 25BCS (11n239/13J360) were sourced from Sigma Aldrich (Cat\#12133C). HSF was harvested from patients undergoing a primary total knee replacement for end-stage osteoarthritis $(n=14)$. Knee joints were sampled to allow suitable volume. Any samples visibly contaminated by haemarthrosis were excluded. There were 6 men and 8 women, average age 73 years (95\% confidence interval: 67.2879.14). The average body mass index (BMI) was 30.22. None of the patients were smokers or vegetarians. Four patients suffered with type II diabetes mellitus. The patient ethnicities were: white Caucasian $(n=7)$, Asian $(n=2)$, black Caribbean $(n=2)$, black African $(n=1)$ and ethnicity not recorded $(n=2)$. Any patient with known communicable disease, pregnant or under 16 years of age was excluded.

\section{Sample preparation and NMR analysis}

Pre-filtered $25 \mathrm{BCS}$ was stored at $-20^{\circ} \mathrm{C}$ until analysis. $\mathrm{HSF}$ was harvested perioperatively and stored at $4^{\circ} \mathrm{C}$ for a maximum of 6 hours before centrifugation (10000g; 15 minutes). The supernatant was decanted, discarding any cells or particulate debris. Samples were then stored for a maximum of 6 months $\left(-80^{\circ} \mathrm{C}\right)$.

Samples $(400 \mu \mathrm{l})$ were combined with buffer $(400 \mu \mathrm{l})$ containing sodium dihydrogen phosphate $(10.64 \mathrm{mg}$ $\mathrm{NaH}_{2} \mathrm{PO}_{4}$ ), sodium azide (400mg NaN 3 ) and 3-(trimethylsilyl)-2,2,3,3-tetradeuteropropionic acid sodium salt in deuterium oxide solution $\left(0.4 \mathrm{mg} \mathrm{TSP} / \mathrm{ml} \mathrm{D}_{2} \mathrm{O}\right)$. Chemicals were purchased from Sigma-Aldrich.

System blanks were run in tandem with biofluids to exclude any contaminants from the collection, reagents, glassware or disposables. 25BCS sample analysis was repeated throughout the analysis to demonstrate quality control (QC) and reproducibility.

\section{NMR data acquisition.}

All experiments were performed using a Bruker ${ }^{\circledR}$ Avance III $600 \mathrm{MHz}$ spectrometer with a Samplejet 96well autosampler (Bruker Biospin ${ }^{\circledR}$, Rheinstetten, Germany). One dimensional ${ }^{1} \mathrm{H}$-NMR experiments were performed at 310K. Samples were analysed using two NMR pulse sequences with water suppression. The NOESYPR1D sequence contains magnetic field gradients in the mixing period, and was performed with a mixing time of $10 \mathrm{~ms}$, a delay between two $90^{\circ} \mathrm{RF}$ pulses of $3 \mu \mathrm{s}$, and a relaxation time between two free induction delays (FID) of 4s. Proteins and large molecules yield poorly resolved bands in the NMR spectrum, whilst small molecules produce sharp peaks. The CPMG sequence had a relaxation time $(\mathrm{D}=$ 
$4 \mathrm{~s})$, spin echo delay $\tau=0.3 \mathrm{~ms}$ and the number of loops $n=128(30)$. This sequence supresses the signals of large molecules, minimising background signal and allowing a clearer assessment of the small molecules. The NOESYPR1D and CPMG methods were acquired with 32 scans and 96,000 data points. The FIDs were multiplied by an exponential function equal to a line broadening factor of $0.3 \mathrm{~Hz}$, a zerofilling producing $128 \mathrm{k}$ data points and followed by Fourier transformation.

NMR spectra were automatically phased and baseline corrected using Topspin ${ }^{\circledR}$ v3.2 software (Bruker) and referenced for chemical shifts to the anomeric ${ }^{1} \mathrm{H}$ peak of a-glucose $(\delta=5.23 \mathrm{ppm})$. Data processing was performed using Matlab ${ }^{\circledR}$ software v9.0.0 (Mathworks ${ }^{\circledR}$, Natick, Massachusetts, USA). Spectra were aligned using a published method to allow peak comparison between samples(31). Spectral intensities were normalised using median-fold change(32).

\section{Metabolite identification}

Metabolite identification was achieved by matching the NMR chemical shifts and peak multiplicities to information held in the literature and metabolite databases: Human Metabolite Database (HMDB) and the Livestock Metabolome Database $(\operatorname{LMDB})(33,34)$. Statistical total correlation spectroscopy (STOCSY) was used to aid metabolite identification by demonstrating peaks which show statistical correlations within the spectra, and thus arise from the same molecule or one functionally related(35).

\section{Statistical analysis.}

Data were transformed and aligned using Matlab® software. Multi-variate linear regression modelling was performed using SIMCA® software v14.1 (MKS Umetrics ${ }^{\mathrm{TM}}$, Umeå, Sweden). The dataset was investigated using an unsupervised principal component analysis (PCA) model, where no class information about the data is used to build the model. This was followed by a supervised orthogonal partial least squares discriminant analysis (OPLS-DA), using pre-defined class information to identify variables discriminating between the classes. The models were evaluated using the $R^{2} X$ and $R^{2} Y$ values, indicating how well the variance is represented. The cross-validation $Q^{2} Y$ metric was used to assess the strength of model prediction. The significance of the metabolic differences between the models was reported using cross-validation p-values. A permutation test was performed 100 times.

An S-line plot identified spectral regions causing the separation between 25BCS and HSF groups. This reports the pCORR, which is a vector of correlations between the scores of the OPLS-DA models and the X data matrix, in this case the spectral intensity at each NMR chemical shift axis defined data point. The results are colour-coded on the loadings of the OPLS-DA models, thereby highlighting spectral regions increased in either 25BCS or HSF. Spectral regions with a pCORR $\geq 0.8$ were investigated further. This watershed was chosen to give $95 \%$ confidence or better(36).

\section{Wear Measurement}

HSF samples with suitable volume to permit further analysis $(n=8)$ and 25BCS stocks $(n=2)$ were used as lubricants in a tribology bench test. Replicate wear measurement was performed upon samples where 
volume allowed; a single sample was tested three times and two samples were tested twice.

The test uses a CoCrMo ball (19.05mm diameter) reciprocating against a CoCrMo disc (Fig. 1). The ball reciprocation replicates joint motion although cross shear is absent. The testing bath chamber was kept at $37^{\circ} \mathrm{C}$ and a thermocouple ensured that contact temperatures were stable and no thermal degradation was seen. The tests were conducted under nitrogen to prevent oxidation.

Tests were run for 1 hour (72000 cycles) and where the fluid volume allowed the test was repeated. The reciprocation of the ball across the disc produces a wear scar on both (Fig. 1). After testing a detergent washed the disc to remove denatured proteins. The wear scar depth on the disc was measured at $30 \%$, $50 \%$ and $70 \%$ along its length (Fig. 1) using a White Light Interferometer (Wyko NT9100). The mean of these measurements provided the average wear scar depth (AWD)(9). If tests were repeated, the mean was compared against the metabolic NMR data. The AWD was correlated with the variation in NMR spectral intensities using a STOCSY analysis. Spectral regions showing a significant linear correlation (Spearman correlation $>0.8$ ) were investigated, thereby indicative of a strong correlation and greater than $95 \%$ confidence( 36,37$)$.

\section{Results}

\section{Observations}

Handling the fluids revealed that osteoarthritic HSF was consistently more viscous than 25BCS. This manifested challenges transferring the HSF samples into narrow aperture NMR tubes.

\section{Quality Control}

Evaluation of the blank samples revealed the presence of contaminant peaks from the reagents used and the laboratory consumables, at very low intensities. These peaks were discarded from any interpretation (Supplementary Table 1). Five consistent metabolites were integrated in the QC sample spectra. Variation of these integrals was consistently less than $2 \%$ (Supplementary Table 2) far below any typical biological variability across the samples.

\section{NMR Evaluation of HSF vs BCS}

The NMR spectra of the two groups were qualitatively assessed. There is a very broad background in the NMR spectra due to major proteins, lipids and complex carbohydrates. Whilst differences between HSF and 25BCS can be observed, little information can be obtained.

The mean NOESYPR1D NMR spectrum of the 25BCS stocks was compared to the mean HSF spectrum and any regions where the mean 25BCS spectrum fell outside the $95 \%$ confidence interval of the HSF spectra were evaluated (Fig. 2 and Table 1).

The profiles of the lipid peaks were similar although some broad bands $(\delta=1.5 \mathrm{ppm}$ and $\delta=2.7 \mathrm{ppm})$ differ. The peak (increased in 25BCS, $\delta=2.7 \mathrm{ppm}$ ) arises from poly-unsaturated fatty acid moieties 
probably incorporated into complex lipids as fatty acyl esters (e.g. phospholipids, glycerolipids, etc). The peak (increased in $\mathrm{HSF}, \delta=1.5 \mathrm{ppm}$ ) reflected $\mathrm{CH}_{2}$ group adjacent to the fatty acyl ester group. HSF lipoproteins are thought to derive from serum and increase with inflammation but provide a smaller contribution to the overall lipid profile(38).

The broad peaks $(\delta=3.0-4.5 \mathrm{ppm})$ are similar in both fluids and are probably due to the carbohydrate moieties of the complex carbohydrates. The peaks near 2.0ppm are due to $\mathrm{N}$-acetyl groups present in the carbohydrate moieties with differences observed.

Sharp, resolved peaks present in the spectra arising from small molecule metabolites, were analysed further using the CPMG NMR spectra. PCA analysis identified small molecule differences between 25BCS and HSF. The PCA scores plot revealed marked separation using the first component between 25BCS and HSF, with an $R^{2} X$ of 0.521 (Fig. 3). Therefore, the overriding variation in the spectra is due to the differences between 25BCS and HSF. The differences originated from a mixture of small molecules peaks and broad background peaks from proteins and other macromolecules (despite CPMG sequences largely supressing macromolecule signals). Variability in the HSF samples clustered into two main groups. Scrutinising the clinical data did not reveal any patient factors to account for this clustering.

An orthogonal partial least squares discriminant analysis (OPLS-DA) model with 25BCS and HSF defined as classes identified significant metabolite differences between them (1 predictive \& 1 orthogonal component, $R^{2} X=0.56, R^{2} Y=0.992, Q^{2} Y=0.959, \mathrm{p}=1.28 \times 10^{-13}$, Fig. 4 with acceptable permutation testing, Fig. 5). The S-line plot highlighted the spectral regions reporting the strength of the distinction, with those data points pCORR $\geq 0.8$ being investigated further (Fig. 6, Table 2).

The 25BCS samples showed greater lactate and creatine content with concurrent reductions in pyruvate and glucose. The terminal $\mathrm{CH}_{3}$ signal of fatty acid chains from lipoproteins was elevated. HSF samples had more amino acids: alanine, glutamine and the branch chain amino acids: leucine and valine. Citrate, acetate and the $\mathrm{N}$-acetyl group of $\mathrm{N}$-acetyl-glycoproteins were also increased. Isopropyl alcohol was seen within the 25BCS samples but absent from the blank samples used to identify contaminants.

\section{Wear Analysis using HSF and BCS fluids}

The NOESY1D spectra of HSF samples produced several spectral regions of high positive correlation to AWD (Fig. 7, Table 3).

The amino acids: lysine, glutamine, glycine, threonine, asparagine, proline, histidine and tyrosine correlated strongly to measured AWD. The correlation to the broad envelope baseline $(\delta=6-6.8 \mathrm{ppm})$ is non-specific and attributed to proteins.

Energy substrates and intermediates glucose and citrate correlated with wear. Multiplet peaks $(\delta=3.938$, $3.975 \mathrm{ppm})$ and a singlet peak $(\delta=7.067 \mathrm{ppm})$ showed a strong correlation but could not be identified. 
The GAG peak $(\delta=7.98 \mathrm{ppm})$ and a shoulder of the $\mathrm{N}$-acetyl peak (previously attributed to proteoglycan breakdown in synovial fluid, $\delta=2.02 \mathrm{ppm}$ ) were universally elevated with increasing AWD(39-42).

The CPMG NMR spectra of the HSF samples failed to show any correlation to the AWD values, either of macromolecules or small molecules, presumably due to macromolecular signal suppression in the CPMG NMR sequence.

\section{Discussion}

This study investigates an important and hitherto unaddressed issue of patient synovial fluid composition and its impact upon implant wear and risk of failure. Furthermore, comparisons were made between patient synovial fluid and the accepted lubricant for validation.

NMR spectroscopy distinguished small molecule differences between HSF and 25BCS. HSF samples contained an altered lipid profile, with lower levels of choline and 3-hydroxyisobutyrate. HSF metabolic products lactate, glucose and creatine were also reduced. Amino acids alanine, valine, glutamine and leucine were elevated.

When correlating the wear testing studies to the NMR spectroscopy of the fluids, significance was detected for both macromolecules and small molecules. However, all wear correlations disappear once the macromolecule signal is suppressed. Therefore, it is possible that these significant small molecules: amino acids (glutamine, glycine, threonine, asparagine, histidine, proline and tyrosine), glucose and citrate are bound to macromolecules.

Correlations to wear were seen with the NMR peaks from GAG and the N-acetyl groups. Prior reports have demonstrated these signals originate from proteoglycan breakdown in synovial fluid(40-44). Therefore, it is hypothesised that a proteoglycan breakdown and possibly GAG is directly correlated to material wear.

\section{Metabolic differences between 25BCS and HSF}

A large variation in osteoarthritic HSF small molecule composition was seen. Previously comparisons had only studied macromolecules(45). The variation in HSF composition demonstrated by the PCA analysis was consistent with other NMR descriptions of the osteoarthritic populations(44).

Synovial fluid is formed from an endocapillary filtrate of blood serum(46). Therefore, HSF and 25BCS fluids are expected to share composition and be different. This is relevant in scenarios of inflammation, like osteoarthritis, when there is increasing capillary wall permeability. Scenarios driving selective capillary wall permeability, active transport to or from the synovial fluid or metabolic processes occurring within the joint itself could cause differences between the fluids(47).

The presence of active joint inflammation reduces the oxygen tension and yields an anaerobic state(48, 49). Animal studies of osteoarthritic synovial fluid compared to healthy controls found an elevation in anaerobic metabolism, fatty acid chain oxidation and proteoglycan degradation(50). The free oxygen of 
bovine and human arterial blood is similar $(51,52)$. Therefore, it was predicted that osteoarthritis HSF samples would be anaerobic compared to a non-diseased 25BCS sample. Despite this, 25BCS samples had consistently higher levels of lactate but lower pyruvate. This may be due to a lack of pyruvate production from glycolysis with concomitant consumption to lactate.

It is possible the industrial processes of harvesting, filtering $(>0.2 \mu \mathrm{m})$, and packaging commercially available 25BCS have ongoing cellular metabolism thereby increasing anaerobic metabolites. However, due to centrifugation prior to storage, HSF purposefully will not. This is so HSF will metabolically mirror the in vivo human joint as best as possible. It was not possible to mimic the processes of 25BCS production in this study.

The HSF samples contained higher concentrations of glucose and creatine. Furthermore, the lipid peaks in 25BCS provided stronger NMR peaks from the $\left(\mathrm{CH}_{2}\right)_{\mathrm{n}}$ moiety $(41)$. Higher levels of choline and 3hydroxyisobutyrate in the 25BCS samples suggest a destruction of complex lipid species and fatty acid metabolism(50). Alternatively, it could indicate a high level of gluconeogenesis.

The presence of proteoglycan in the HSF is supported, although not confirmed, by the observation of the $\mathrm{N}$-acetyl moiety $(2.03 \mathrm{ppm})$. A larger $\mathrm{N}$-acetyl signal has been indicative of proteoglycan destruction, including hyaluronan(50). Free amino acids alanine, glutamine, leucine and valine were elevated in the HSF compared to the 25BCS model, possibly due to proteinolysis. Changes in valine have been reported due to prolonged low temperature storage, but it remains unclear if this is the case(53).

Isopropyl alcohol was found within the 25BCS samples. This contaminant has been seen from skin swabbing but the presence within the 25BCS cannot be explained. The effect upon lubrication is not known.

The 25BCS model fluid contained more acidic anaerobic metabolites: lactate, pyruvate and 3hydroxybutyrate. Macromolecule surface binding of lipid and hyaluronan has been shown to be effective in reducing implant wear $(54,55)$. It is possible that the acid metabolites could affect the surface binding of charged species, protein conformation or protein aggregation, thereby limiting lubrication. In addition, the acidic species could propagate corrosion wear of the implant(56). Whilst synovial fluid contains an inherent buffering capacity, the measurement of the $\mathrm{pH}$ before and after wear testing has shown the potential for disturbance with a consistent increased alkalinity(9).

\section{The correlation between implant wear and the metabolic profile}

When testing wear using HSF and relating the AWD back to the fluid composition by NOESYPR1D NMR spectroscopy, several small and macromolecules were significant. However, the CPMG spectra universally showed no significance, presumably due to the suppression of macromolecule signals. The loss of significant small molecules when changing from the NOESYPR1D spectra to the CPMG implies that small molecules are bound or complexed in fast exchange with macromolecules. This effect has been 
described in plasma with binding to human serum albumin and the potential exists within HSF due to its large proteoglycan and GAG content(57).

Several amino acids positively correlated with measured CoCrMo wear but their change in response to joint disease is known to be variable. The design of this sudy was limited by the use of HSF only from OA patients with no control samples available. The reports of these metabolites in synovial fluid suggest that their detection decreases with osteoarthritis, although, glycine, asparagine, proline and tyrosine are reported to increase. There are no reports of changing levels of lysine or histidine with joint disease in HSF. The significance of these metabolites is unclear. Cartilage NMR spectroscopic studies have suggested alteration of glycine levels correlating with proteoglycan destruction and collagen destruction(50).

The presence of significant N-acetyl $(\delta=2.02 \mathrm{ppm})$ and GAG $(\delta=7.98 \mathrm{ppm})$ peaks indicates that GAG plays a role in lubrication of this specific wear test. HSF is known to contain several GAG molecules including: chondroitin sulphate, keratan sulphate and hyaluronan. It has been postulated that these molecules provide lubrication in the native joint; hyaluronan joint supplementation by injection being an accepted osteoarthritis treatment(58-60). It is indicated that one shoulder of the N-acetyl peak $(\delta=$ $2.02 \mathrm{ppm}$ ) is significant, rather than the whole peak, suggesting a specific contributor. It is not possible using these methods to specifically identify which proteoglycan species is relating to CoCrMo wear. However, it seems clear that GAG macromolecule destruction is responsible.

The significance of increasing glucose, citrate and pyruvate with CoCrMo wear may be related to metabolic stress and changing energy utilisation within the samples due to joint disease. The level of glucose has been shown to both increase and decrease in joint disease $(50,61)$. In addition, citrate and pyruvate have also been demonstrated to consistently increase with joint disease and this change was attributed to anaerobic metabolism(50). Whilst these metabolites alter in joint disease and relate directly to metabolic stress, it is unclear what the correlation with prosthetic wear is attributable to. It is possible that these metabolites may confound with another lubricating component of HSF, increased in metabolic stress and/or advanced joint disease. Alternatively, the anaerobic environment of the joint may have a direct relationship with the lubrication properties of the HSF due to alteration of the $\mathrm{pH}$ or molecular interactions. However, this seems unlikely since the other detectable anaerobic metabolic markers such as lactate and formate were not significant.

An alternative hypothesis is that the glucose and citrate may be bound to a significant lubricating macromolecule within the HSF likely to be protein and GAG in origin as evidenced by our findings.

\section{Future Studies}

We aim to study the macromolecular structure of the significant products that play a key role in implant wear. In addition, we aim to substitute alternative bearing surfaces such as metal-on-poly, ceramic-onceramic or ceramic-on-poly, to investigate if the same metabolites are key to measured wear. 


\section{Conclusion}

In conclusion we have illustrated the small molecule and wear differences between HSF and its lubricant model 25BCS, raising questions as to the validity of 25BCS as a surrogate model for HSF in implant wear testing. The study also suggested that key metabolites, including destruction of the GAG molecule, correlated with increased implant wear with in vitro testing, specifically with a high glucose citrate, lysine, glycine, tyrosine and proline content. This will serve as a guide to the development of more representative model fluids in the future and further research into targeting synovial fluid to preserve prosthetic joints.

\section{Clinical Implications}

This is the first study to demonstrate metabolites which linearly correlate with wear on implant surfaces. This raises the tantalising prospect of whether metabolic supplementation may promote implant longevity. Furthermore, an understanding of the metabolic composition of synovial fluid may yield information about optimal implant selection or predicted wear, promoting the possibility for personalised medicine.

\section{Abbreviations}

Human Synovial Fluid (HSF), Nuclear Magnetic Resonance (NMR), 25\% wt Bovine Calf Serum (25BCS), Cobalt Chromium Molybdenum (CoCrMo), Glycosaminoglycans (GAG), Carr-Purcell-Meiboom-Gill (CPMG), Body Mass Index (BMI), Quality Control (QC), Nuclear Overhauser effect spectroscopy (NOESY1D), free induction decays (FIDs), Human Metabolite Database (HMDB), Livestock Metabolome Database (LMDB), Statistical total correlation spectroscopy (STOCSY), principal component analysis (PCA), orthogonal partial least squares discriminant analysis (OPLS-DA), correlation to OPLS-DA scores (pCORR), average wear scar depth (AWD)

\section{Declarations}

\section{Ethics Approval and Consent to Participate}

Ethical approval was granted (NRES Committee London, Chelsea, UK. 12/05/2015). The study was performed in accordance with the ethical standards in the 1964 Declaration of Helsinki. All patients consented to the study.

\section{Consent for Publication}

All patients consented to publication as a part of the consent process to be enrolled in the study 


\section{Availability of Data and Materials}

The dataset(s) supporting the conclusions of this article is available in the US National Library of Medicine repository, [www.clinicaltrials.gov - NCT02565160].

\section{Competing Interests}

No competing interests to disclose.

\section{Funding}

The work was supported by the National Institute for Health Research (NIHR) Imperial Biomedical Research Centre (BRC) and the Department of Orthopaedics at Imperial College Healthcare NHS Trust (ICHNT).

\section{Author Contributions}

All authors contributed substantially to the conception and design of the work. MKJJ, PA, UV and HS contributed to sample collection and assembly of data. MKJ, GG \& CLB were involved in the statistical analysis. MKJJ, CMG and PC drafted the article. RB, HRTW, JCL, CMG and PC assisted with interpretation of the data, arranged logistical and funding support and critically reviewed the manuscript before final approval. All authors had full access to all the data and take responsibility for the integrity of the data and the accuracy of the data analysis.

\section{Acknowledgements}

We thank Metabometrix Ltd for provision of expertise and resource, Professor Justin Cobb and the Musculoskeletal laboratory, Imperial College London for administrative support and the provision of laboratory facilities and Professor Keith Jaggard for his comments and guidance.

\section{References}

1. National Joint Registry 14th Annual Report. Herfortshire, UK: National Joint Registry for England and Wales, Northern Island and the Isle of Man, 2016.

2. Pacha-Olivenza MA, García-Alonso MC, Tejero R, Escudero ML, Gallardo Moreno AM, GonzálezMartín ML. Microbiologically induced corrosion of titanium implants. Orthopaedic Proceedings. 2017;99-B(SUPP_1):22-.

3. Saikko V. A multidirectional motion pin-on-disk wear test method for prosthetic joint materials. J Biomed Mater Res. 1998;41(1):58-64. 
4. Medley JB, Krygier JJ, Bobyn JD, Chan FW, Lippincott A, Tanzer M. Kinematics of theMATCO@ hip simulator and issues related to wear testing of metal-metal implants. Proc Instn Mech Engrs. 1997;211(H):89-99.

5. Standardization, IOf. ISO 14242-1:2014; Implants for surgery - Wear of total hip-joint prostheses Part 1: Loading and displacement parameters for wear-testing machines and corresponding environmental conditions for test 2014 [Available from: https://www.iso.org/obp/ui/\#iso:std:63073:en.

6. Martin-Alarcon L, Schmidt TA. Rheological effects of macromolecular interactions in synovial fluid. Biorheology. 2016;53(2):49-67.

7. Dowson D, Hardaker $C$, Flett $M$, Isaac GH. A hip joint simulator study of the performance of metal-onmetal joints: Part I: The role of materials. J Arthroplasty. 2004;19(8 Supplement):118-23.

8. Mavraki A, Cann PM. Friction and lubricant film thickness measurements on simulated synovial fluids. Proceedings of the Institution of Mechanical Engineers, Part J: Journal of Engineering Tribology. 2009;223(3):325 - 35.

9. Stevenson H, Parkes M, Austin L, Jaggard MKJ, Akhbari P, Vaghela U, et al. The development of a small-scale wear test for CoCrMo specimens with human synovial fluid. Biotribology. 2018:1-10.

10. Bowsher J, Hussain A, Williams P, Shelton J. Metal-on-metal hip simulator study. of increased wear particle surface area due to. 'severe'patient activity. Proceedings of the Institution of Mechanical Engineering. 2006;220(2):279 - 87.

11. Smith SL, Dowson D, Goldsmith AA. The effect of femoral head diameter upon lubrication and wear of metal-on-metal total hip replacements. Proceedings of the Institution of Mechanical Engineers. 2001;215(2):161 - 70.

12. Hui AY, McCarty WJ, Masuda K, Firestein GS, Sah RL. A systems biology approach to synovial joint lubrication in health, injury, and disease. Wiley Interdiscip Rev Syst Biol Med. 2012;4:15-37.

13. Board NE. 2018 15th Annual Report: National Joint Registry for England, Wales,Northern Ireland and the Isle of Man. London, United Kingdom: 2018.

14. Glyn-Jones S, Pandit H, Kwon Y-M, Doll H, Gill HS, Murray DW. Risk factors for inflammatory pseudotumour formation following hip resurfacing. The Journal of Bone Joint Surgery British volume. 2009;91-B(12):1566-74.

15. Kitano T, Ateshian GA, Mow VC, Kadoya Y, Yamano Y. Constituents and pH changes in protein rich hyaluronan solutions affect the biotribological properties of artificial articular joints. J Biomech. 2001;34:1031-7.

16. Mazzucco D, Scott R, Spector M. Composition of joint fluid in patients undergoing total knee replacement and revision arthroplasty: correlation with flow properties. Biomaterials. 2004;25(18):4433-45.

17. Fuchs B, Bondzio A, Wagner U, Schiller J. Phospholipid compositions of sera and synovial fluids from dog, human and horse: a comparison by 31P-NMR and MALDI-TOF MS. J Anim Physiol Anim Nutr. 2009;93:410-22. 
18. Parkes M. Lubrication mechanisms of lipids and proteins in model synovial fluids. Imperial College London; 2014.

19. Liao Y-S, Benya PD, McKellop HA. Effect of Protein Lubrication on the Wear Properties of Materials for Prosthetic Joints. J Biomed Mater Res. 1999;48:465-73.

20. Saikko V., Tribol., J. Effect of lubricant protein concentration on the wear of ultra-high molecular weight polyethylene sliding against a CoCr counterface. J Tribol. 2003;125:638-42.

21. Sawae $Y$, Yamamoto $A$, Murakami T. Influence of protein and lipid concentration of the test lubricant on the wear of ultra high molecular weight polyethylene. Tribol Int. 2008;41:648-56.

22. Wang A, Essner A, Schmidig G. The Effects of Lubricant Composition on in vitro Wear Testing of Polymeric Acetabular Components. J Biomed Mater Res. 2003;68B:45-52.

23. Lindon JC, Holmes E, Nicholson JK. So what's the deal with metabonomics? Anal Chem. 2003;75:384A-91A.

24. Odunsi K, Wollman R, Ambrosone C, Hutson A, McCann S, Tammela J, et al. Detection of epithelial ovarian cancer using ${ }^{1} \mathrm{H}$-NMR-based metabonomics. Int J Cancer. 2005;113:782-8.

25. Wang Z, Chen Z, Yang S, Wang Y, Yu L, Zhang B, et al. (1)H NMR-based metabolomic analysis for identifying serum biomarkers to evaluate methotrexate treatment in patients with early rheumatoid arthritis. Exp Ther Med. 2012;4:165-71.

26. Yu B, Zheng Y, Nettleton JA, Alexander D, Coresh J, Boerwinkle E. Serum metabolomic profiling and incident CKD among African Americans. Clin J Am Soc Nephrol. 2014;9(8):1410-7.

27. Athersuch TJ. The role of metabolomics in characterizing the human exposome. Bioanalysis. 2012;4(18):2207-12.

28. Hong J, Yang L, Zhang D, Shi J. Plant Metabolomics: An Indispensable System Biology Tool for Plant Science. Int J Mol Sci. 2016;17(6).

29. Nicholson JK, Lindon JC. Systems biology: Metabonomics. Nature. 2008;455(7216):1054-6.

30. Lindon JC, Nicholson JK. Spectroscopic and Statistical Techniques for Information Recovery in Metabonomics and Metabolomics. Annual Review of Analytical Chemistry. 2008;1(1):45-69.

31. Veselkov KA, Lindon JC, Ebbels TMD, Crockford D, Volynkin VV, Holmes E, et al. Recursive SegmentWise Peak Alignment of Biological H-1 NMR Spectra for Improved Metabolic Biomarker Recovery. Anal Chem. 2009;81:56-66.

32. Veselkov KA, Vingara LK, Masson P, Robinette SL, Want E, Li JV, et al. Optimized preprocessing of ultra-performance liquid chromatography/mass spectrometry urinary metabolic profiles for improved information recovery. Anal Chem. 2011;83(15):5864-72.

33. Goldansaz SA, Guo AC, Sajed T, Steele MA, Plastow GS, Wishart DS. Livestock metabolomics and the livestock metabolome: A systematic review. PLoS One. 2017;12(5):e0177675.

34. Wishart DS, Jewison T, Guo AC, Wilson M, Knox C, Liu Y, et al. HMDB 3.0-The Human Metabolome Database in 2013. Nucleic Acids Res. 2013;41(Database issue):D801-7. 
35. Cloarec O, Dumas M-E, Craig A, Barton RH, Trygg J, Hudson J, et al. Statistical Total Correlation Spectroscopy: An Exploratory Approach for Latent Biomarker Identification from Metabolic $1 \mathrm{H}$ NMR Data Sets. Anal Chem. 2005;77(5):1282-9.

36. Rummel RJ. Applied Factor Analysis. Evanston: Northwestern University Press; 1970.

37. Mukaka MM. Statistics corner: A guide to appropriate use of correlation coefficient in medical research. Malawi medical journal: the journal of Medical Association of Malawi. 2012;24(3):69-71.

38. Oliviero F, Lo Nigro A, Bernardi D, Giunco S, Baldo G, Scanu A, et al. A comparative study of serum and synovial fluid lipoprotein levels in patients with various arthritides. Clin Chim Acta. 2012;413(12):303-7.

39. Albert K, Michele S, Guenther U, Fial M, Gall H, Saal J. ${ }^{13} \mathrm{C}$ NMR investigation of synovial fluids. Magn Reson Med. 1993;30:236 - 40.

40. Damyanovich AZ, Staples JR, Chan ADM, Marshall KW. Comparative Study of Normal and Osteoarthritic Canine Synovial Fluid using $500 \mathrm{MHz}{ }^{1} \mathrm{H}$ Magnetic Resonance Spectroscopy. Journal of Orthpaedic Research. 1999;17:223-31.

41. Damyanovich AZ, Staples JR, Marshall KW. ${ }^{1} \mathrm{H}$ NMR investigation of changes in the metabolic profile of synovial fluid in bilateral canine ostroarthritis with unilateral joint denervation. Osteoarthritis Cartilage. 1999;7:165-72.

42. Lacitignola L, Fanizzi FP, Francioso E. al. e. $1 \mathrm{H}$ NMR investigation of normal and osteoarthritic synovial fluid in the horse. Vet Comp Orthop Traumatol. 2008;21:85-8.

43. Duffy JM, Grimshaw J, Guthrie DJ, McNally GM, Mollan RA, Spedding PL, et al. $1 \mathrm{H}$-nuclear magnetic resonance studies of human synovial fluid in arthritic disease states as an aid to confirming metabolic activity in the synovial cavity. Clin Sci. 1993;85(3):343-51.

44. Zhang W, Sun G, Likhodii S, Liu M, Aref-Eshghi E, Harper PE, et al. Metabolomic analysis of human plasma reveals that arginine is depleted in knee osteoarthritis patients. Osteoarthritis Cartilage. 2016;24(5):827-34.

45. Balazs EA, Watson D, Duff IF, Roseman S. Hyaluronic Acid in Synovial Fluid. I. Molecular Parameters of Hyaluronic Acid in Normal and Arthritic Human Fluids. Arthritis Rheum. 2005;10(4):357-76.

46. McCarty WJ, Luan A, Siddiqui M, Hansen BC, Masuda K, Sah RL. Biomechanical properties of mixtures of blood and synovial fluid. Journal of orthopaedic research: official publication of the Orthopaedic Research Society. 2011;29(2):240-6.

47. Kushner I, Somerville JA. Permeability of human synovial membrane to plasma proteins. Relationship to molecular size and inflammation. Arthritis Rheum. 1971;14(5):560-70.

48. Treuhaft PS, McCarty DJ. Synovial fluid pH, lactate, oxygen and carbon dioxide partial pressure in various joint diseases. Arthritis Rheumatology. 1971;14:475-84.

49. Lund-Olsen K. Oxygen tension in synovial fluid. Arthritis Rheumatology. 1970;13:769-76.

50. Jaggard MKJ, Boulange CL, Akhbari P, Vaghela U, Bhattacharya R, Williams HRT, et al. A systematic review of the small molecule studies of osteoarthritis using nuclear magnetic resonance and mass 
spectroscopy. Osteoarthritis Cartilage. 2019;27(4):560-70.

51. MSD and the Veterinary Manual Kenilworth, NJ USA. Sharp M. \& Dohme Corp; 2016 [Available from: http://www.msdvetmanual.com/appendixes/reference-guides/blood-gas-reference-ranges.

52. McAuley D. Common Laboratory (LAB) Values - ABGs New York, USA: UBM Media LLC; 2000 [Available from: http://www.globalrph.com/abg_analysis.htm.

53. Damyanovich AZ, Staples RJ, Marshall KW. The effects of freeze/thawing on human synovial fluid observed by $500 \mathrm{MHz} 1 \mathrm{H}$ magnetic resonance spectroscopy. J Rheumatol. 2000;27(3):746-52.

54. Bruck AL, Karuppiah KS, Sundararajan S, Wang J, Lin Z. Friction and wear behavior of ultrahigh molecular weight polyethylene as a function of crystallinity in the presence of the phospholipid dipalmitoyl phosphatidylcholine. J Biomed Mater Res B Appl Biomater. 2010;93(2):351-8.

55. Zhang M, Pare P, King R, James SP. A novel ultra high molecular weight polyethylene-hyaluronan microcomposite for use in total joint replacements. II. Mechanical and tribological property evaluation. J Biomed Mater Res A. 2007;82(1):18-26.

56. Willert HG, Broback LG, Buchhorn GH, Jensen PH, Koster G, Lang I, et al. Crevice corrosion of cemented titanium allow stems in total hip replacements. Clin Orthop Relat Res. 1996;333:51-75.

57. Jupin M, Michiels PJ, Girard FC, Spraul M, Wijmenga SS. NMR identification of endogenous metabolites interacting with fatted and non-fatted human serum albumin in blood plasma: Fatty acids influence the HSA-metabolite interaction. J Magn Reson. 2013;228:81-94.

58. Henderson EB, Grootveld M, Farrell A, Smith EC, Thompson PW, Blake DR. A pathological role for damaged hyaluronan in synovitis. Ann Rheum Dis. 1991;50(3):196-200.

59. Bellamy N, Campbell J, Robinson V, Gee T, Bourne R. WG. Viscosupplementation for the treatment of osteoarthritis of the knee. Cochrane Database Syst Rev. 2005;2:CD005321.

60. Lo GH, LaValley M, McAlindon TFDT. Intra-articular hyaluronic acid in treatment of knee osteoarthritis: a metaanalysis. JAMA. 2003;290:3115-21.

61. Sitton NG, Dixon JS, Bird HA, Wright V. Serum and synovial fluid histidine: a comparison in rheumatoid arthritis and osteoarthritis. Rheumatol Int. 1986;6:251-4.

\section{Tables}

Table 1

NOESYGPPR1D NMR spectral regions that distinguish between HSF and BCS fluids from an OPLSDA model 


\begin{tabular}{|c|c|c|c|}
\hline $\begin{array}{l}\text { Chemical Shift } \\
\text { (ppm) }\end{array}$ & Peak type & Elevated & Peak Assignment (HMDB \& STOCSY) \\
\hline $0.358-0.401$ & $\begin{array}{l}\text { Broad background } \\
\text { peaks }\end{array}$ & BCS & Not assignable \\
\hline $0.46-0.56$ & $\begin{array}{l}\text { Broad background } \\
\text { peaks }\end{array}$ & BCS & Not assignable \\
\hline $0.575-0.76$ & $\begin{array}{l}\text { Broad background } \\
\text { peaks }\end{array}$ & BCS & Not assignable \\
\hline 0.76 & $\begin{array}{l}\text { Broad background } \\
\text { peaks }\end{array}$ & BCS & Not assignable \\
\hline 0.975 & Doublet & HSF & Valine \\
\hline 1.03 & Doublet & HSF & Valine \\
\hline 1.16 & Doublet & BCS & Isopropyl alcohol \\
\hline 1.325 & Doublet & BCS & Lactate \\
\hline 1.46 & Doublet & HSF & Alanine \\
\hline $1.49-1.54$ & $\begin{array}{l}\text { Broad background } \\
\text { peaks }\end{array}$ & HSF & \\
\hline 1.72 & Triplet & HSF & Lysine \\
\hline 1.91 & Singlet & HSF & Acetic Acid \\
\hline 1.996 & Singlet & BCS & Lipid species $\left(\mathrm{CH}_{2}-\mathrm{C}=\mathrm{C}\right)$ \\
\hline 2.039 & Singlet & HSF & N-acetyl glycoproteins \\
\hline 2.12 & Multplet & HSF & Glutamine \\
\hline 2.365 & Singlet & HSF & Pyruvate \\
\hline 2.45 & Mulitplet & HSF & Glutamine \\
\hline 2.51 & Doublet & HSF & Citrate \\
\hline 2.65 & Singlet & HSF & $\begin{array}{l}\text { Unknown (related to triplet of doublet - } \\
2.338 \mathrm{ppm} \text { ) }\end{array}$ \\
\hline $2.78-2.96$ & $\begin{array}{l}\text { Broad background } \\
\text { peaks }\end{array}$ & HSF & Not assignable \\
\hline 3.03 & Singlet & BCS & Creatine \\
\hline 3.21 & Singlet & HSF & Choline \\
\hline
\end{tabular}




\begin{tabular}{|llll|}
\hline 3.23 & Doublet of doublets & HSF & Glucose \\
\hline 3.35 & Doublet & HSF & Unknown (related to Citrate) \\
\hline 3.92 & Multiplet & HSF & Glucose \\
\hline 4.01 & Multiplet & BCS & Unknown \\
\hline $5.1-5.14$ & Multiplet & BCS & Isopropyl alcohol \\
\hline $5.33-5.7$ & $\begin{array}{l}\text { Broad background } \\
\text { peaks }\end{array}$ & BCS & Not assignable \\
\hline $6.09-6.15$ & $\begin{array}{l}\text { Broad background } \\
\text { peaks }\end{array}$ & BCS & Not assignable \\
\hline 7.05 & $\begin{array}{l}\text { Broad background } \\
\text { peaks }\end{array}$ & BCS & Not assignable \\
\hline $7.4-7.78$ & $\begin{array}{l}\text { Broad background } \\
\text { peaks }\end{array}$ & HSF & Not assignable \\
\hline 7.74 & $\begin{array}{l}\text { Broad background } \\
\text { peaks }\end{array}$ & HSF & Not assignable \\
\hline $8.38-8.64$ & $\begin{array}{l}\text { Singlet } \\
\text { Broad background } \\
\text { peaks }\end{array}$ & BCS & Not assignable \\
\hline $8.86-$ Broad background & BCS & Not assignable \\
\hline
\end{tabular}

Table 2

CPMG NMR spectral regions that distinguish between HSF and BCS fluids from an OPLS-DA model 


\begin{tabular}{|c|c|c|c|c|}
\hline $\begin{array}{l}\text { Chemical shift } \\
\text { (ppm) }\end{array}$ & Peak Type & Elevated & Peak ID (HMDB) & $\mathrm{P}(\mathrm{CORR})$ \\
\hline 0.85 & Broad & BCS & Terminal $\mathrm{CH}_{3}$-LDL/VLDL & 0.986 \\
\hline 1.16 & Doublet & BCS & Isopropyl alcohol & 0.995 \\
\hline 1.33 & Doublet & BCS & Lactate & 0.988 \\
\hline 1.99 & Singlet & BCS & Lipid chain species $\left(\mathrm{CH}_{2}-\mathrm{CH}=\mathrm{C}\right)$ & 0.978 \\
\hline 2.04 & Singlet & HSF & N-acetyl glycoproteins & 0.983 \\
\hline 2.11 & Multiplet & HSF & Glutamine & 0.940 \\
\hline 2.34 & Multiplet & BCS & Glutamate & 0.968 \\
\hline 2.37 & Doublet & HSF & Pyruvate & 0.949 \\
\hline 2.40 & Singlet & BCS & Unknown & 0.987 \\
\hline 2.45 & Quartuplet & HSF & Glutamine & 0.940 \\
\hline 2.52 & Doublet & HSF & Citrate & 0.930 \\
\hline 2.66 & Doublet & HSF & Citrate & 0.938 \\
\hline 2.74 & Broad & BCS & $\begin{array}{l}\text { Lipid chain species }\left(\mathrm{C}=\mathrm{CH}-\mathrm{CH}_{2}-\right. \\
\mathrm{CH}=\mathrm{C})\end{array}$ & 0.959 \\
\hline 3.04 & Singlet & BCS & Creatine & 0.838 \\
\hline 3.19 & Singlet & BCS & Choline & 0.976 \\
\hline 3.23 & $\begin{array}{l}\text { Doublet of } \\
\text { doublets }\end{array}$ & HSF & Glucose & 0.972 \\
\hline 3.45 & Quartet & HSF & Glucose & 0.938 \\
\hline 3.74 & Singlet & HSF & Glucose & 0.989 \\
\hline 3.83 & Singlet & HSF & Glucose & 0.995 \\
\hline 3.85 & Doublet & HSF & Glucose & 0.994 \\
\hline 3.89 & Doublet & HSF & Glucose & 0.985 \\
\hline 3.92 & Singlet & BCS & Creatine & 0.987 \\
\hline 3.99 & Singlet & BCS & Unknown & 0.991 \\
\hline 4.00 & Doublet & BCS & Unknown & 0.992 \\
\hline 4.01 & Multiplet & BCS & Isopropyl alcohol & 0.993 \\
\hline 4.11 & Quartet & BCS & Lactate & 0.989 \\
\hline
\end{tabular}


Table 3

HSF metabolites identified by NOESYGPPR1D NMR spectroscopy that correlate (Spearman >0.8) with AWD or wear 


\begin{tabular}{|c|c|c|c|}
\hline Metabolite ID & Chemical Shift (ppm) & & $\begin{array}{l}\text { Spearman } \\
\text { correlation }\end{array}$ \\
\hline N-Acetyl (GAG) & $2.02(\mathrm{~s})$ & & 0.827 \\
\hline Glutamine & $2.12(\mathrm{~m})$ & $2.44(\mathrm{~m})$ & $0.815 ; 0.863$ \\
\hline Pyruvate & $2.367(\mathrm{~s})$ & & 0.845 \\
\hline Citrate & $2.517(\mathrm{dd})$ & & 0.815 \\
\hline Asparagine & $2.879(\mathrm{~m})$ & & 0.90 \\
\hline Lysine & $3.02(\mathrm{~m})$ & & 0.93 \\
\hline Unknown & Broad baseline peaks 3.09-3.18 & & 0.961 \\
\hline Proline & $3.33(\mathrm{~m})$ & & 0.924 \\
\hline Glucose & $3.4(\mathrm{~m})$ & & 0.948 \\
\hline Glycine & $3.56(\mathrm{~s})$ & & 0.815 \\
\hline Unknown & $3.938(\mathrm{~m})$ & & 0.851 \\
\hline Unknown & Broad baseline peak 4.031-4.046 & & 0.966 \\
\hline Unknown & Broad baseline peak 4.164-4.187 & & 0.973 \\
\hline Threonine & $4.22(\mathrm{~m})$ & & 0.906 \\
\hline $\begin{array}{l}\text { Glycosaminoglycans } \\
\text { (GAG) }\end{array}$ & $4.45(\mathrm{~s})$ & & 0.875 \\
\hline $\begin{array}{l}\text { Glycosaminoglycans } \\
\text { (GAG) }\end{array}$ & $5.907(\mathrm{~m})$ & & 0.809 \\
\hline Proteins $-\mathrm{NH}$ & Broad background peaks 6.07 & & 0.918 \\
\hline Proteins $-\mathrm{NH}$ & $\begin{array}{l}\text { Broad background peaks } 6.229 \text { - } \\
6.254\end{array}$ & & 0.888 \\
\hline Proteins $-\mathrm{NH}$ & $\begin{array}{l}\text { Broad background peaks } 6.377 \text { - } \\
6.454\end{array}$ & & 0.948 \\
\hline Proteins $-\mathrm{NH}$ & $\begin{array}{l}\text { Broad background peaks } 6.568- \\
6.662\end{array}$ & & 0.906 \\
\hline Proteins $-\mathrm{NH}$ & $\begin{array}{l}\text { Broad background peaks } 6.785- \\
6.804\end{array}$ & & 0.827 \\
\hline Proteins -NH & $\begin{array}{l}\text { Broad background peaks 3.783- } \\
6.804\end{array}$ & & 0.809 \\
\hline Tyrosine & $6.889(\mathrm{~m})$ & & 0.827 \\
\hline Histidine & $7.052(\mathrm{~s})$ & $\begin{array}{l}7.795 \\
(d)\end{array}$ & $0.863 ; 0.852$ \\
\hline
\end{tabular}




\begin{tabular}{|lll|}
\hline Proteins $-\mathrm{NH}$ & $\begin{array}{l}\text { Broad background peaks 7.473- } \\
7.781\end{array}$ & 0.918 \\
\hline $\begin{array}{l}\text { Glycosaminoglycans } \\
\text { (GAG) }\end{array}$ & $7.981(\mathrm{~s})$ & 0.888 \\
\hline
\end{tabular}

\section{Figures}
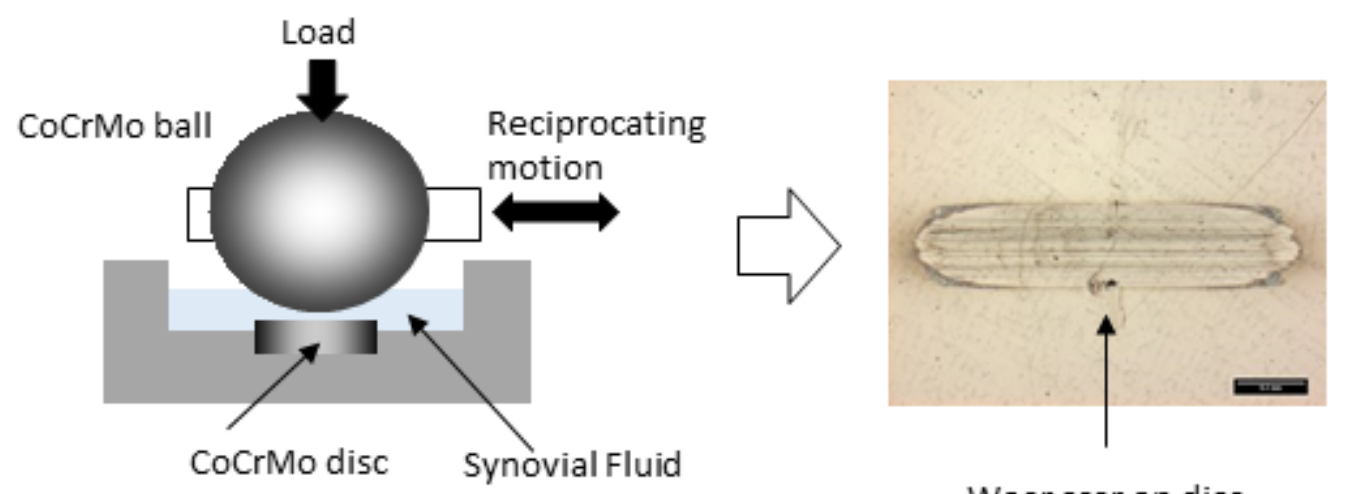

Wear scar on disc
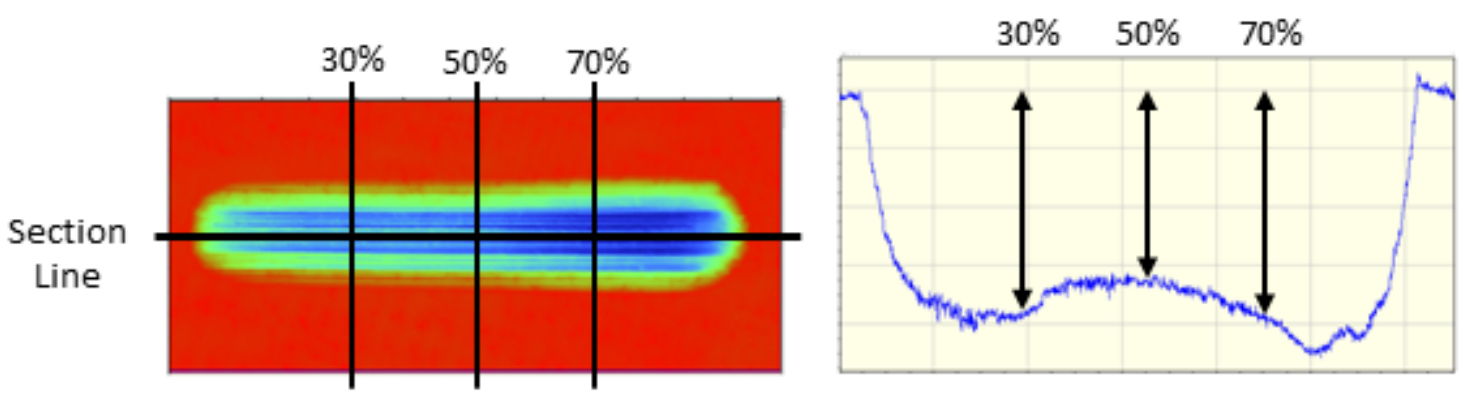

Figure 1

Schematic representation of the biofluid lubricant testing rig using an oscillation motion of a test ball upon a test disc and the subsequent wear scar (upper portion). The subsequent wear scar is measured by averaging the depth at three consistent points along its length (30\%,50\% and $70 \%$; lower portion). 

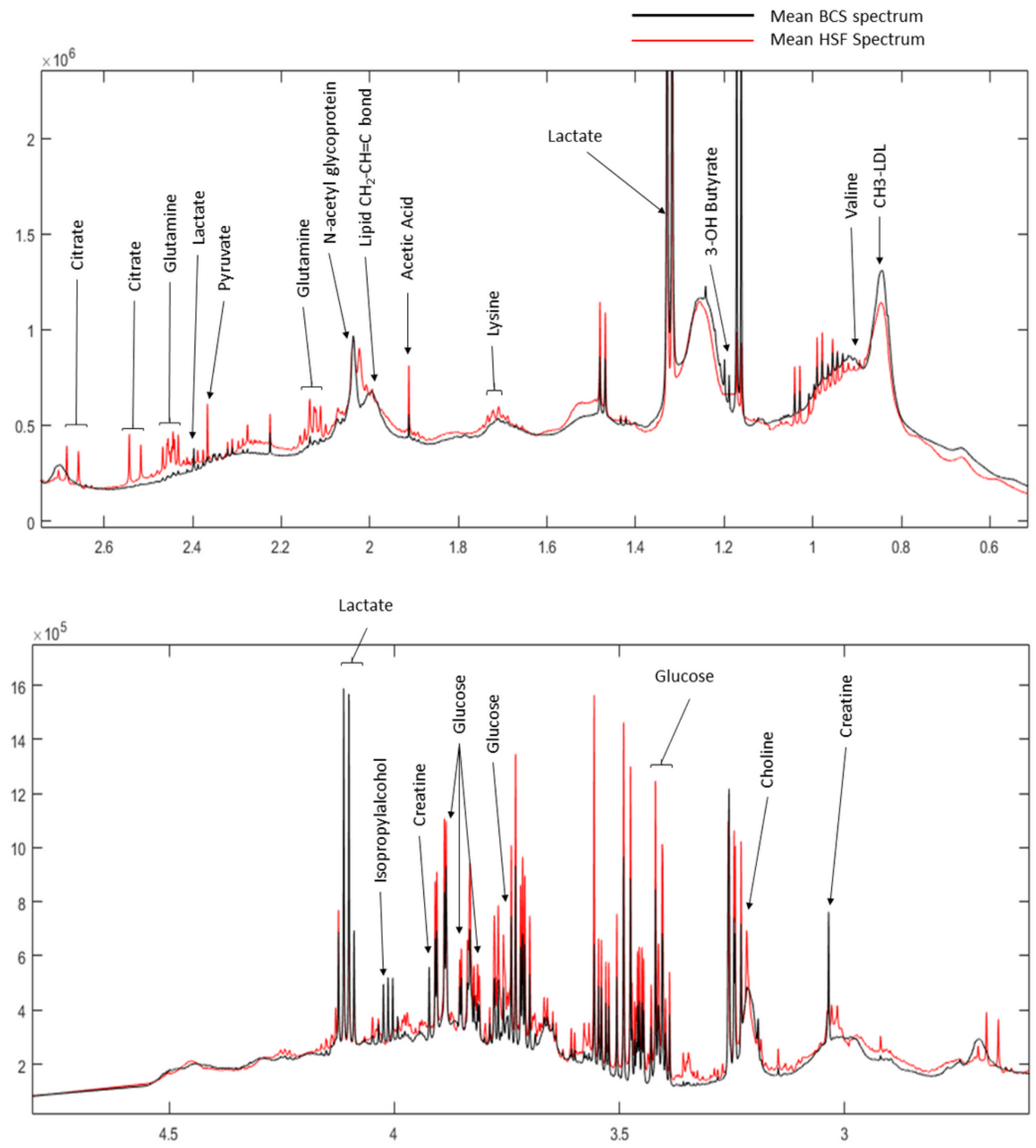

Figure 2

Annotated mean NOESYPR1D NMR spectra and comparison of HSF and 25BCS samples (upper 02.8ppm, lower 2.5-5ppm). The 95\% confidence intervals for the HSF samples are shown in the Supplementary Material and only peaks outside this range were investigated further. 


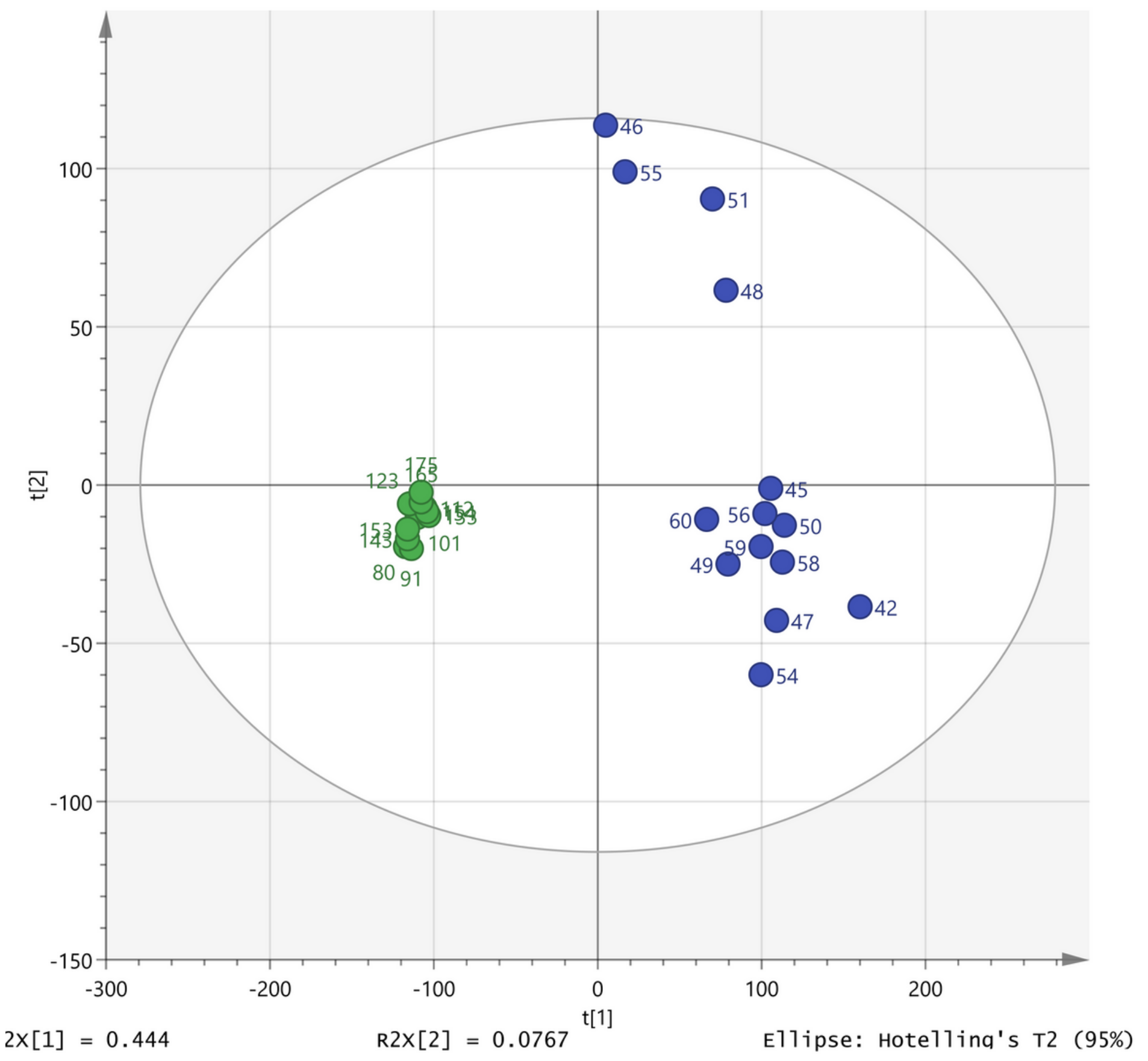

Figure 3

PCA scores plot of the first two principal components comparing CPMG NMR spectra of 25BCS model (green) and HSF samples (blue). (PC1 = 44.4\%, PC2 = 7.67\%). 


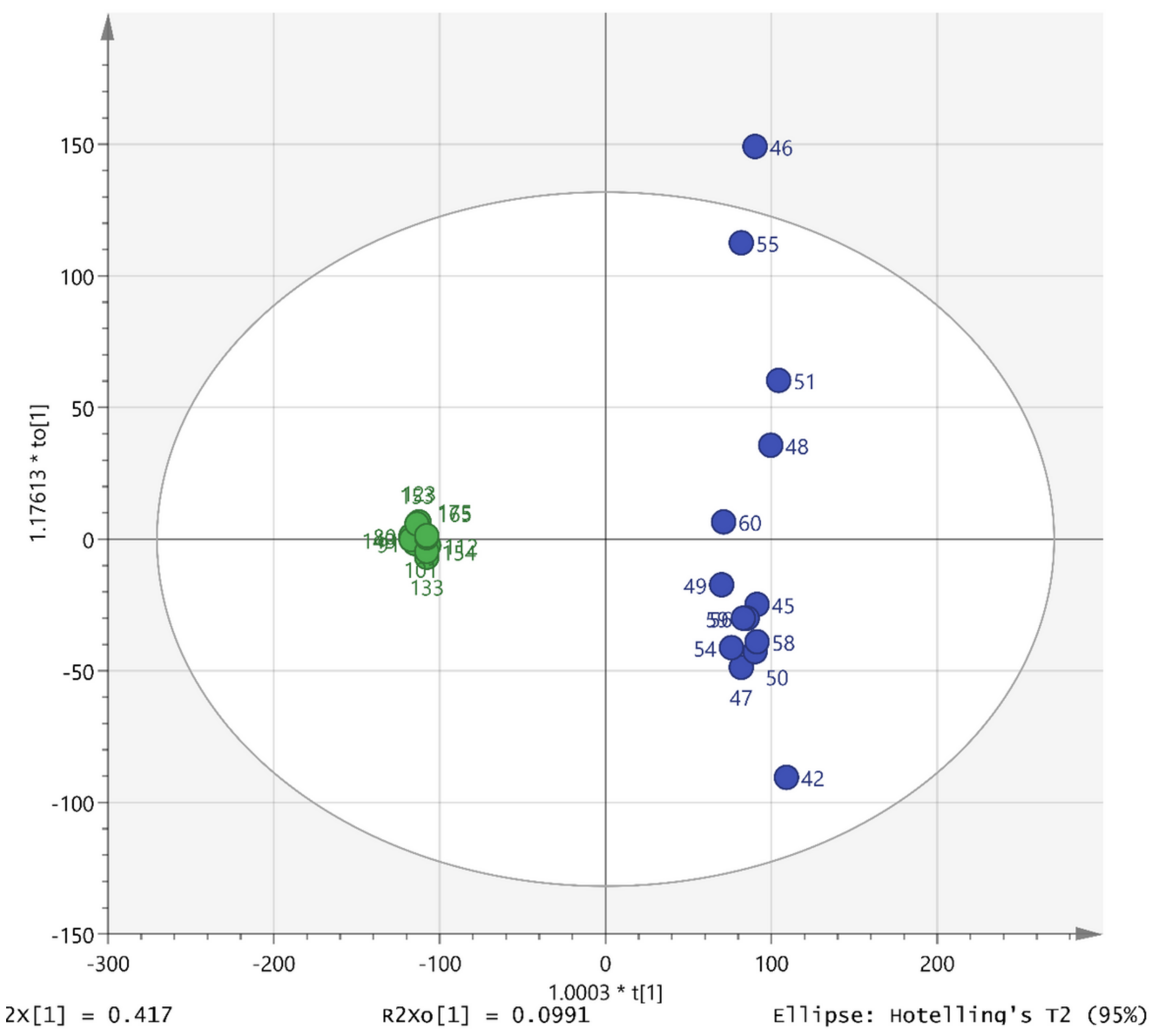

\section{Figure 4}

OPLSDA scores plot of the first two principal components comparing CPMG NMR spectra of BCS model (green) and HSF samples (blue). (PC1 = 41.7\%, PC2 = 9.9\%). 


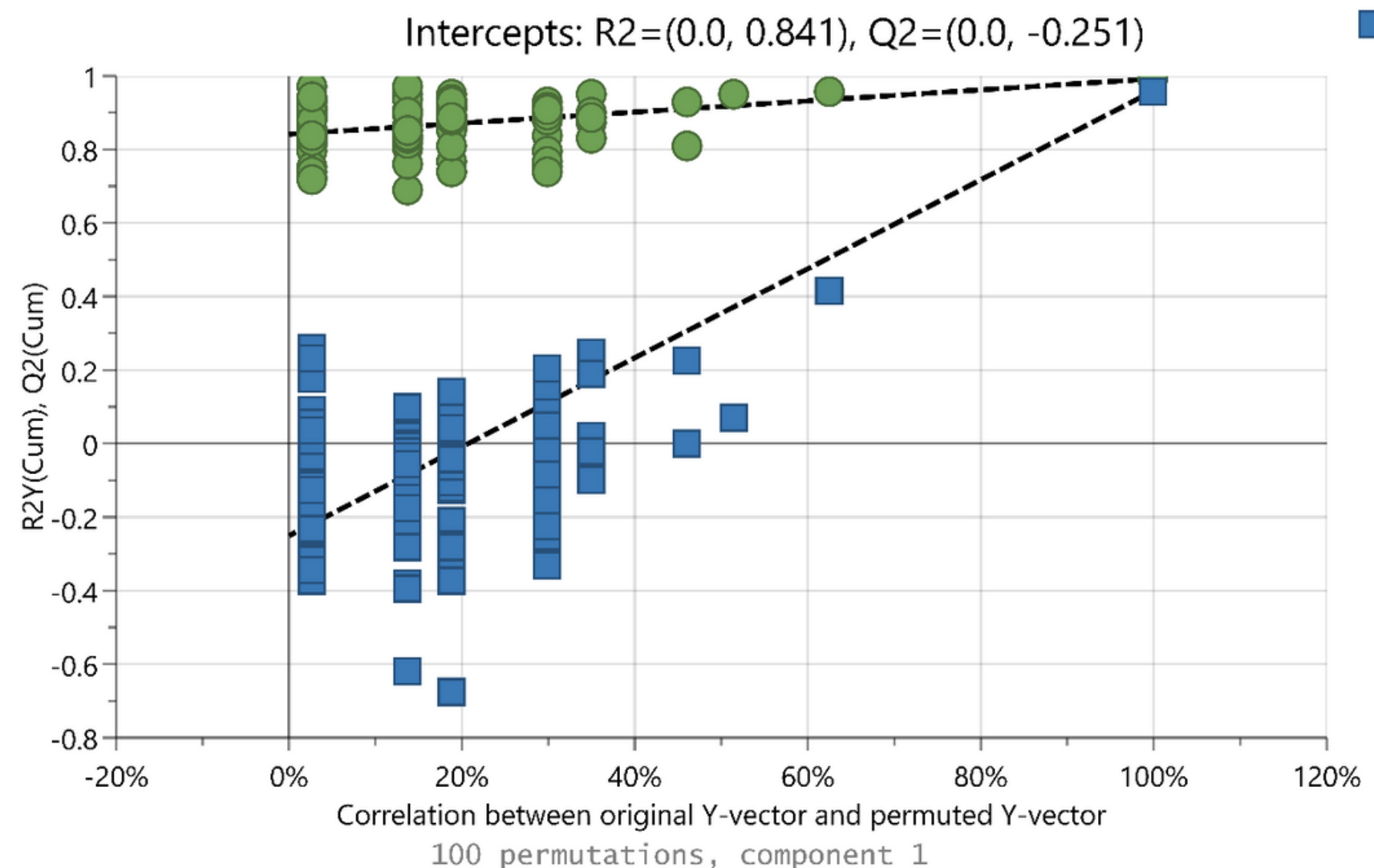

\section{Figure 5}

Permutation testing for the OPLS-DA model analysis of CMPG NMR spectra comparing BCS to HSF 


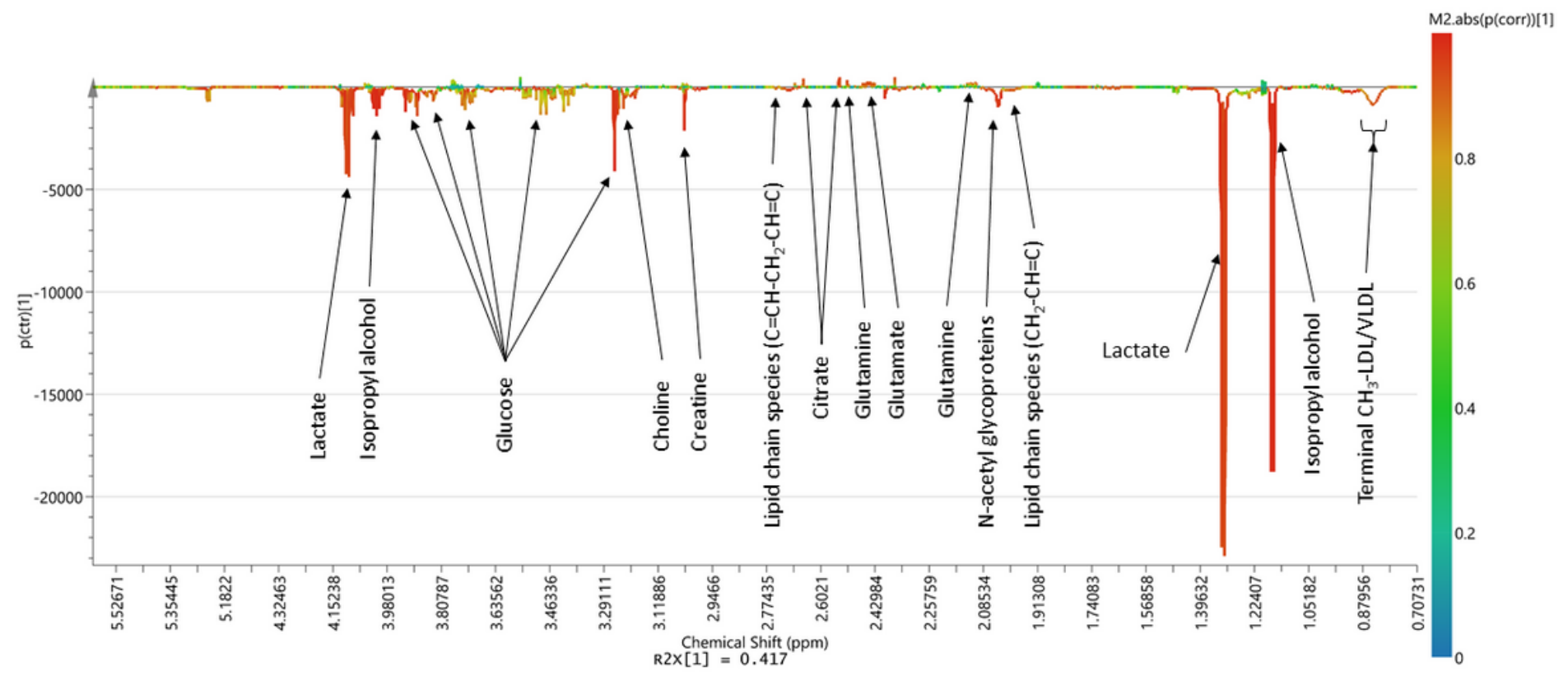

\section{Figure 6}

S-line plot for CPMG NMR spectra after applying an OPLS-DA model to compare HSF and 25BCS groups. The pCORR (variables correlated with the OPLS-DA scores) is represented according to a temperature colour scale. Hotter colours represent the spectral regions with high correlation and thus are responsible for the separation between the HSF and 25BCS groups in the OPLS-DA model. 
NOESY NMR Data STOCSY correlation with the measured Average Wear Depth (AWD)

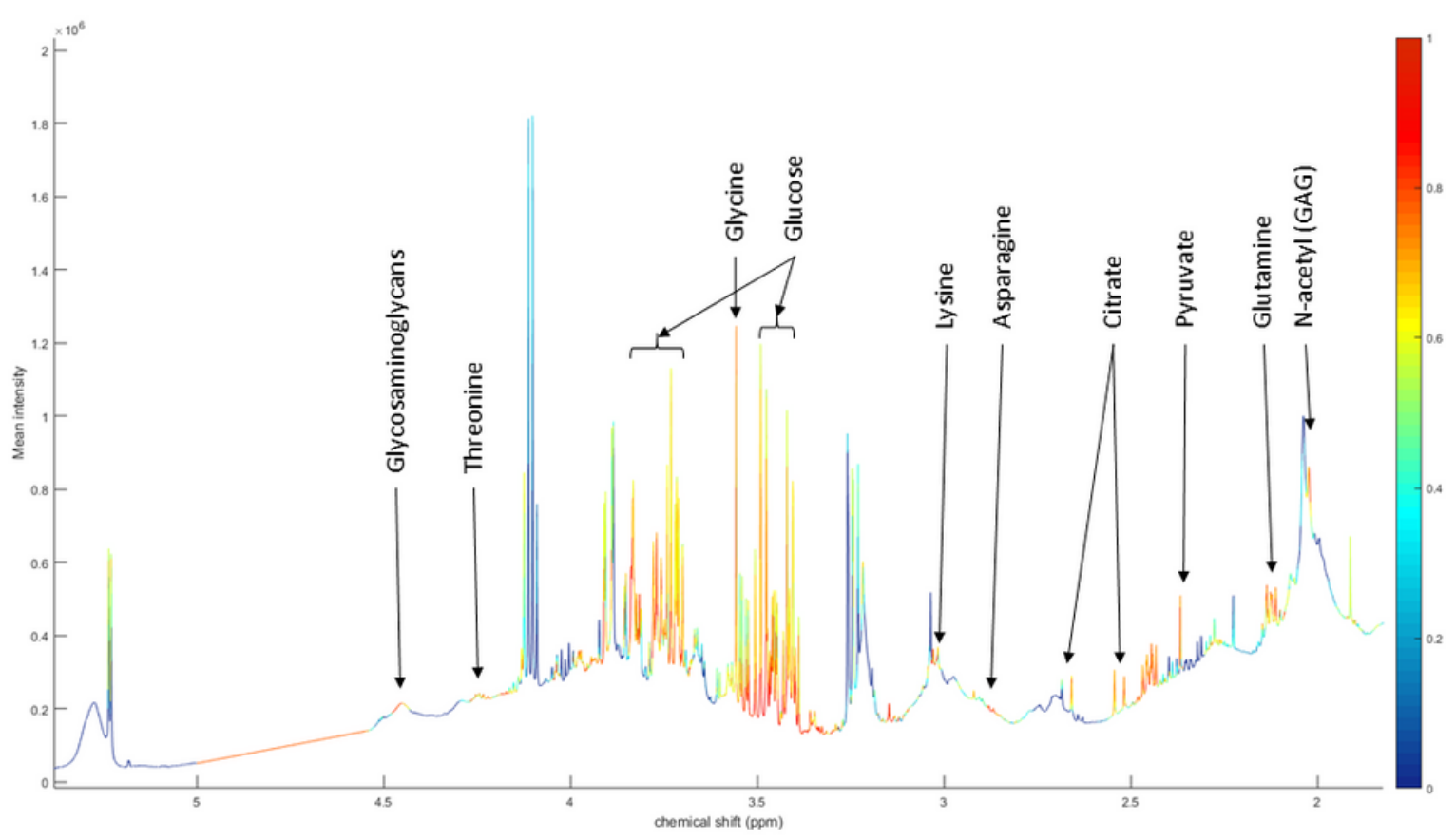

\section{Figure 7}

The mean NOESYPR1D spectrum of the HSF samples depicting areas of correlation to wear by colour. Spearman correlation is represented according to a temperature colour scale. Hotter colours represent the spectral regions with high correlation and correlation to the AWD.

\section{Supplementary Files}

This is a list of supplementary files associated with this preprint. Click to download.

- SupplementaryTable1.docx

- SupplementaryTable2.docx

- SupplementaryTable3.docx 